\title{
Fundamentos de la explicación en ciencias sociales: revisitando el constructivismo de Niklas Luhmann (20 años después)
}

\author{
Foundations for the social sciences explanation: \\ Revisiting NiklasLuhmann's contructivism (20 years later) \\ Jorge Gibert Galassi*; Juan Pablo Venables** \\ "Universidad de Valparaíso, Chile \\ jorge.gibert@uv.cl \\ **Universidad Austral de Chile, Chile \\ jpvenables@uach.cl
}

\begin{abstract}
Resumen
En este trabajo se sostiene que uno de los fundamentos de la teoría de sistemas sociales de Niklas Luhmann, a saber, que no posee ontología, impediría elaborar explicaciones en sociología. Debido a que la teoría posee un fundamento de carácter lógico-formal, que no presupone una ontología, existen obstáculos insalvables para materializar ese objetivo. Por ello, es válido preguntarse por el mérito de una teoría que, después de 20 años, insiste en no alinearse con premisas ontológicas de modo robusto y sugiere, por tanto, un rechazo a integrar a la sociología dentro del conjunto de las ciencias fácticas. Sin embargo, pensamos que la riqueza de la obra luhmanniana permite dialogar con el mainstream de la práctica teórica contemporánea. Se revalidan de este análisis tres reglas sobre lo que "no debe hacerse" para fundamentar filosóficamente una teoría en sociología y una sugerencia clave para superar el problema de la explicación en la sociología de Luhmann: la ciencia social busca y orienta su actividad hacia el descubrimiento y uso de distinciones sustantivas, reales, que permitan enriquecer, también, el contenido fáctico de los enunciados sobre la realidad social.
\end{abstract}

Palabras Claves: sociología, teoría de sistemas sociales, explicación, ontología, autopoiesis.

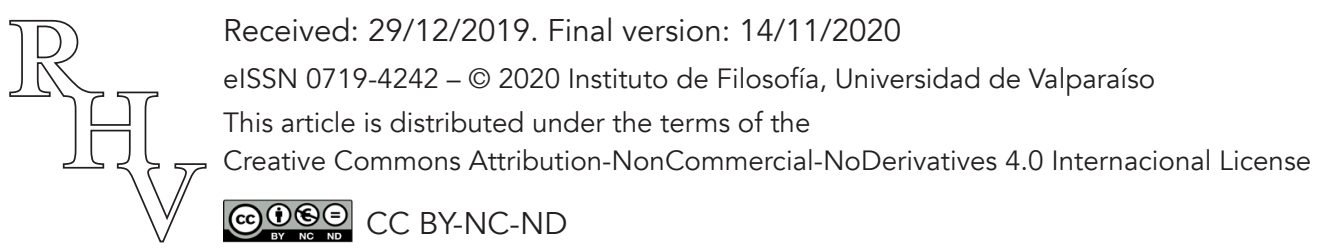


Fundamentos de la explicación en ciencias sociales: revisitando el constructivismo de Niklas Luhmann (20 años después)

Jorge Gibert Galassi; Juan Pablo Venables

\begin{abstract}
In this work, it is argued that one of the foundations of NiklasLuhmann's theory of social systems, namely that it has no ontology, would prevent the elaboration of explanations in sociology. Because the theory has a formal-logical foundation, which does not presuppose ontology, there are insurmountable obstacles to achieving this objective. For this reason, it is valid to wonder about the merit of a theory that, after 20 years, insists on not aligning itself with ontological premises in a robust way and suggests, therefore, a refusal to integrate sociology within the set of factual sciences. However, we think that the richness of Luhmann's work allows a dialogue with the mainstream of contemporary theoretical practice. Three rules about what "should not be done" are revalidated from this analysis to philosophically base a theory in sociology and a key suggestion to overcome the problem of explanation in Luhmann's sociology: social science seeks and directs its activity towards discovery and the use of substantive, real distinctions that also enrich the factual content of statements about social reality.
\end{abstract}

Keywords: sociology, social systems theory, explanation, ontology, autopoiesis.

\title{
1. Introducción
}

En este trabajo se discuten los fundamentos de la explicación en ciencias sociales en un diálogo con la teoría constructivista de Niklas Luhmann ${ }^{1}$. En un sentido secundario, aspira a proponer una alternativa a las dificultades de este empeño.

En el enfoque tradicional, la explicación se vincula al conocimiento, cuando éste último es definido como "creencia verdadera justificada". Se supone que las explicaciones (de)mostrarán que algo es verdadero porque hay buenas razones para creerlo.

Para las ciencias sociales, el dilema explicativo surge del momento que J.S. Mill plantea que es posible una ciencia de la naturaleza humana, dedicada a investigar las conexiones causales entre pensamientos, sentimientos y acciones; pero la define como inexacta debido a la complejidad inherente a la conducta humana $(1882,1026$ y ss.) La "inexactitud" se puede interpretar como la incapacidad de las teorías sociales para predecir los fenómenos de los que se ocupa. Esto significa que, si no hay predicción, no hay explicación; pues desde un punto de vista ampliamente divulgado (Hempel 1988), la diferencia entre explicar y predecir es pragmática o de secuencia: si podemos explicar, podemos predecir. Y viceversa.

Pero la complejidad de la sociedad dificulta llegar a explicaciones acotadas, dado que la mayoría de los fenómenos sociales son multicausales. En general, las ciencias sociales

\footnotetext{
${ }^{1}$ No es el objetivo de este trabajo resumir, en ningún sentido y bajo ningún respecto, la obra sociológica de Luhmann, cuya extensión y variedad es notable.
}

Revista de Humanidades de Valparaíso, 2020, No 16, 191-214

(c) $(i) \Theta(9)$ CC BY-NC-ND 
se han apoyado en una concepción de legalidad amplia, la Ley de Hecho (Bunge 1974), que puede expresarse de la siguiente manera: es un enunciado hipotético que ha sido confirmado empíricamente respecto de una relación aproximadamente constante (causal), bajo ciertos parámetros, entre dos o más variables. En la tradición de las ciencias sociales podemos encontrar varios ejemplos, como la distribución paretiana de las rentas, que según Laurent (1908) fue la aportación más importante en la historia de la economía matemática.

La incapacidad de predecir eventos da argumentos a quienes sostienen la tesis que es imposible la explicación en ciencias sociales. En particular, cuando estos eventos son de gran envergadura, trascendencia y nocivos para la humanidad, tales como guerras, crisis económicas o conflictos étnicos. Sin embargo, hay argumentos para refutar esa idea. En primer lugar, porque si las ciencias sociales son ciencias fácticas, aspiran como toda ciencia a explicar los fenómenos de los que se ocupa. En segundo lugar, porque es una tesis yerma que conduce a una práctica epistémica acientífica o anticientífica. Y finalmente, porque es falsa (empíricamente): hay explicaciones por doquier (Bunge 1983; 1997; 1999; Fallding 1976; Joseph y Kennedy 2000; Turner 1991; Wallace 1983; etc.).

Cabe señalar que no todas las explicaciones comparten la misma fisonomía. La práctica convencional de la investigación social constata la relación estadística entre personas con múltiples atributos. Otra práctica consiste en atribuir causalidad a los "motivos-porque" y los "motivos-para" de las personas (Schütz y Luckmann 1973), o bien analizar la intencionalidad desde la teoría de juegos (Elster 1984). Hoy en día, la denominada sociología analítica prefiere reemplazar la pregunta "porqué ocurrió eso" por el concepto de "mecanismo" (Demeulenaere 2011) y algunas versiones del "emergentismo" (Padgetty Powell 2012), de modo que los ribetes del debate sobre la explicación siguen vigentes.

La teoría de Luhmann tuvo el mérito de replantear el modo en que se elaboraba teoría, con consecuencias relevantes para la explicación en ciencias sociales. Desde nuestra modernidad digital, es plausible pensar que la sociología clásica enfatizó características propias de sociedades menos complejas, donde el individuo o la acción podían tener un lugar destacado en el mueblaje ontológico de la teoría social. O bien, que una determinada organización, como el Estado, hegemonizara la forma de explicar la reproducción social. En ese sentido, el abordaje de Luhmann responde también a nuestras preocupaciones: ¿cómo concebir las explicaciones en la teoría sociológica de modo que capture el atributo de creciente intangibilidad y diferenciación de los fenómenos sociales en la actual modernidad? Ello nos conduce a la discusión sobre los fundamentos de la explicación en la teoría de Luhmann. El núcleo del trabajo se centra en la novedad del planteamiento luhmanniano, la necesidad de omitir la discusión ontológica en la construcción teórica y, a nuestro juicio, su consecuencia inesperada, que consiste en postular un método improductivo para tal construcción. Con todo, se afirma que la obra de Luhmann, al procesar de modo nutricio la tradición sociológica, permite revisitarla desde nuevas claves interpretativas. 
Así, primero discutiremos los aspectos críticos de su propuesta sobre los fundamentos, básicamente, la discusión sobre ontología y método de construcción teórica. Seguidamente, discutiremos algunos conceptos centrales que dificultan la explicación sociológica acorde a la teoría luhmanniana. En tercer lugar, seleccionamos algunos aspectos que consideramos promisorios para, revisitados, generar un diálogo fructífero con las explicaciones sociológicas de teorías que presuponen ontologías y finalmente, sugerimos una conclusión.

\section{Ontología y método de construcción teórica}

En su obra, Luhmann define la discusión ontológica como una discusión sobre una categoría. Como tal, no remite a la discusión sobre lo que representa, es decir, entidades reales. La razón es que, para el autor, el programa al que adhiere -el constructivismo- sostiene que la realidad siempre permanece desconocida. En efecto, en su texto The cognitive program of constructivism and a reality that remains unknown, plantea que las cuestiones epistemológicas son problemáticas, ya sea porque existen factores sociales que influyen en todo conocimiento y hacen pensar que se funda en la "convención" o como resultado de una "negociación", o bien porque hay determinada evidencia a favor de que la cognición es una operación que sucede sin contacto directo con el mundo exterior. De allí que Luhmann infiere que "conocer es solo un proceso autorreferencial" (Luhmann 1990,437) ${ }^{2}$. La consecuencia de ello es que, para Luhmann, "la ontología no es un rompecabezas perenne que deba resolverse de nuevo, sino una categoría históricamente determinada para ser descartada" (Rasch 2013, 38). O al decir de Rodríguez: "la sociología clásica contenía demasiada ontología. Era la fiel heredera del pensamiento vétero europeo y, por lo mismo, sus conceptos centrales no permitían ver la realidad de una sociedad como la moderna" (2006, VIII).

Al instalar la discusión en ese plano, constatamos que se abren dos opciones de construcción teórica y ninguna encara las implicaciones de este "descarte" ontológico. La primera es la histórica semántica y justifica la segunda, que es metodológica o procedimental.

En la primera opción, la interpretativa histórica, Luhmann afirma que "en el siglo XVII aumentan los indicios de que el código moral se desontologiza" (2006, 312), aludiendo a que el código prescinde de la identidad entre lo moral y lo social basado en la autoridad religiosa de una sociedad simple. La virtud solo es posible debido al vicio. Pero, a fin de cuentas, esto implica la desaparición de la moral como atributo de un individuo, y si la moral no requiere del individuo, entonces la moral se reintegra a la sociedad como código binario bondad-maldad, que es la distinción cómo funciona el sistema social moral en la

${ }^{2}$ Todas las citas entre comillas de los textos en inglés son traducciones nuestras.

Revista de Humanidades de Valparaíso, 2020, No 16, 191-214

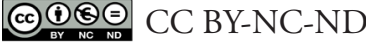


sociedad. La semántica histórica sugiere que toda ontología es un relato que, en el caso de las sociedades estratificadas, puede ser usado; pero en el caso de la sociedad moderna, no (Bryson 2018, 218).

A nuestro juicio, esto es erradicar la ontología de la discusión de modo ilegítimo. Podemos completar la ontología, pues el mueblaje del mundo cambia en la medida que la especie humana construye la realidad social y la transforma a lo largo de la historia (Searle 1995). Pero no podemos anular la ontología o validarla teóricamente como mera semántica. Eso es confundir la entidad con el concepto. Solo le resta concluir que "el conocimiento solo puede conocerse a sí mismo, aunque puede, como por el rabillo del ojo determinar que esto solo es posible si hay algo más que cognición" (Luhmann 1990, 437, cursivas nuestras).Esto evidencia la ambigüedad, que va desde la negación de un mundo allá afuera a una deslucida apertura que concluye en polisemia. En su estilo: "la cognición se ocupa de un mundo externo que permanece desconocido y, como resultado, viene a ver que no se puede ver lo que no se puede ver" (Luhmann 1990, 437). Ciertamente, el carácter incompleto y perfectible del conocimiento (o la rueda del conocimiento-ignorancia que moviliza la investigación científica) no implica una postura escéptica radical.

Dado que su teoría no posee anclaje ontológico, el punto de partida es que el mundo debe dejarse afuera, incluidos las personas/individuos, para centrarse en el concepto de sistema. Es decir, una representación cibernética de la sociedad. La pregunta relevante para Luhmann no es cómo está compuesto el sistema, cuál es su estructura y cuáles elementos posee. Su pregunta es cómo cada sistema social podría reconocer y procesar la información sobre ese mundo y esos individuos, dado que la sociedad moderna (su perspectiva histórica-semántica para erradicar la ontología) es "un sistema policontextural que permite un sinnúmero de descripciones acerca de su complejidad" (Luhmann 2006, 21-22). Pero la complejidad es un constructo teórico-matemático que, cuando se trata de representaciones pertenecientes a las ciencias fácticas (genes, estrellas o posteos de Twitter) implican ontologías específicas (Gibert 2006). Una de las objeciones de Luhmann sobre incluir al individuo como parte de la sociedad, es que dicha incorporación "entraría en oposición evidente con el concepto de derechos humanos, en especial con el de igualdad" (Luhmann 2006, 16), pues significaría el diseño de una teoría de la diferenciación 2 social que clasifica $-\mathrm{y}$ discrimina- a los seres humanos. En cambio, suponer que los seres humanos están fuera del sistema social, implica un trato humanista o neutral ceteris paribus.

Esto nos lleva a preguntarnos si acaso las comunicaciones son el fundamento ontológico de la teoría luhmanniana. En efecto, dado que "sólo las comunicaciones y todas las comunicaciones contribuyen a la autopoiesis de la sociedad" (Luhmann 2006, 64), podríamos pensarlo. Como la comunicación es producida por actores a los que el mundo se les aparece con diferentes significados subjetivos, con Tada $(2020,10)$, se podría postular que el giro epistemológico de Luhmann en la teoría macro sociológica puede considerarse como una adaptación de la sociología fenomenológica de Schütz y Natanson (1962), con su tesis de las múltiples realidades. Sin embargo, la comunicación es sentido hecho 
praxis y como postula el autor, el sentido es el medio por el cual un sistema social adopta una determinada diferencia con su entorno, pues la recursividad de la autopoiesis de la sociedad está organizada "reflexivamente; es decir, mediante la aplicación de la comunicación a la comunicación" (Luhmann 2006, 105). Esto significa, para su teoría, que las comunicaciones no son elementos de una determinada estructura real (como podrían serlo para una teoría con premisas ontológicas), sino un dispositivo sistémico de constitución del sistema mismo.

En su afán desontologizador, Luhmann está intentando hablar siempre desde un plano teórico que representa un manual de funcionamiento, donde no hay elementos sino sólo operaciones. En ese sentido, es una teoría epistémica, un modus operandi. Al erradicar la ontología, debe centrar su fundamento de construcción teórica en la teoría de la forma, que reemplaza la distinción sujeto-objeto por la distinción adentro/afuera; sistema/entorno; identidad/diferencia. Se plantea que el mundo es incognoscible con independencia del observador, para lo cual avanza una caracterización de cómo opera ese observador.

En la segunda opción de construcción teórica sin ontología, la operación de observación constituye la clave. Ella es posible debido a que la observación misma es una metodología o procedimiento. Así, nos encontramos con el rol de lo lógico-formal en su diseño teórico. Según Rodríguez, se trata de radicalizar el método funcional "entendiendo función en el sentido lógico-matemático del término, vale decir, como un esquema lógico regulador que permita comparar entre sí, como equivalentes funcionales, sucesos que desde otra perspectiva serían absolutamente incomparables" (2005, XII). Si la sociología luhmanniana es una sociología del suceso (contingente), el método para caracterizarlo es la distinción entre sistema y entorno que el sistema hace. El suceso (ser/no-ser) es la unidad analítica. La pregunta metodológica es por la distinción sistema/entorno, un caso particular de aplicación del cálculo general de las formas. Dice Luhmann: "apoyándonos levemente en Spencer Brown podría distinguirse el lado interior de la forma - en calidad de atractor de la operación - de su lado exterior. Operar en el modo del sentido significa, pues, que todas las operaciones tienen actualmente lugar en el lado interior de la forma (o no lo tienen); pero para eso, es necesario el otro lado de la forma" (2006, 35).

En la práctica de investigación social, habitualmente el método está subordinado al objeto. Como en Luhmann no hay "objetos específicos", entonces, la descripción se subordina al método. Bajo este procedimiento, la descripción de sistemas es siempre una autodescripción de su propia distinción entre sistema/entorno y "representa al mundo en la medida en que su otro lado carga con aquello que todavía no ha sido señalado" (Luhmann 2006, 38). Pero para ser efectiva, debe darse la "re-entry de una distinción en lo distinguido mediante ella. La diferencia sistema/entorno se da dos veces: como distinción producida por el sistema y como distinción observada en el sistema" (Luhmann 2006, 46). El raciocinio es que no existe ningún problema epistemológico, pues estamos frente a operaciones. No hay indexación ni elementos. "El sistema se coloca a sí mismo en la 
situación de oscilar entre operaciones evaluadas como positivas o negativas, y en la de oscilar entre autorreferencia y heterorreferencia. El sistema se confronta con un futuro indeterminable para él mismo" (Luhmann 2006, 28-29).

Nuevamente, al rechazar la posibilidad de una descripción desde afuera, Luhmann especula y deja éste "unmarkedspace" al libre arbitrio de cualquier interpretación. De hecho, la distinción entre lo autorreferido y lo heterorreferido (re-entry) implica una suerte de aceptación del mundo exterior. Pero ¿qué implica ese "afuera” desde el cual se produce la re-entry? En palabras de Luhmann:

[...] la distinción ser/no-ser como distinción fundante (primary distinction) se reemplaza por la distinción dentro/fuera (o autorreferencia/heterorreferencia) del observador, porque, según esta nueva versión, antes de que un observador pueda emplear la distinción ser/no-ser, debe estar producido como observador. $(2006,722)$.

En otras palabras, el método es conveniente, toda vez que se juega por marcar un lado de la distinción, pero si se equivoca, puede apelar a que el otro lado está incluido en la distinción misma. Así, la teoría desontologizada de Luhmann aparece como un artilugio, fundado en la plasticidad arbitraria de que:

no hay entonces una diferencia primordial entre el ser y el no-ser...El mundo mismo es más bien incalificable en relación con positivo/negativo. Por eso precisamente puede (y debe) distinguirse cuando se pretende señalar algo; o dicho con otras palabras, una distinción no niega lo que no señala; por el contrario, lo presupone como 'unmarkedspace' $(2006,171)$.

El uso de esto es controversial, toda vez que la ciencia factual se basa en la identidad y no en la diferencia, como lo plantea Luhmann. Esto es debido a que cualquier afirmación debe responder -empírica o hipotéticamente- a un esquema causal y no a una contingencia indeterminada. En un ejemplo cualquiera, e.g., Teoría del contrato, el objeto de la teoría es el contrato y no el "no-contrato". En efecto, podemos decir: A causa B, siendo A los "factores que acarrean, o concurren hacia, el establecimiento de un contrato" y B el "Contrato" en sí. Mediante la operación lógica del modus tollens, podemos afirmar que si A causa $\mathrm{B}$, entonces, ante la negación de $\mathrm{B}(\sim \mathrm{B})$, la conclusión es la negación de $\mathrm{A}(\sim \mathrm{A})$. $\mathrm{Si} \sim \mathrm{B}$ es "Contrato", ¿qué sabemos, entonces, del fenómeno del "No-Contrato"? A lo menos, que su existencia se debería en primera instancia a la inexistencia de los "factores que acarrean el contrato", $\sim$ A. Por el contrario, ¿podemos afirmar algo? La respuesta es negativa, puesto que el dominio barrido por la negación es mayor que el de la afirmación. Como $\sim \mathrm{A}$ es cualquier cosa que no sea $\mathrm{A}$, entonces la explicación del establecimiento del contrato resulta, lógicamente, indeterminada.

Esta indeterminación responde al hecho que la teoría de Luhmann elaboró "semánticas" sobre el fenómeno, en vez de teorías con un fundamento ontológico. Con su método, 
nuestro autor fusiona el legado del funcionalismo parsoniano, la teoría de las formas de Spencer-Brown y la teoría de la autopoiesis de Maturana y Varela (1995). Pero el pegamento de esto, al no tener un fundamento ontológico, queda en la ambigüedad. Reemplaza la acción con la comunicación, viendo en esta una cosa y no un atributo de (producido por) personas, organizaciones o instituciones. Como indica Rasch:

[...] muestra cómo la relación entre epistemología y la ontología se basa en una paradoja. Por un lado, la reflexión en la modernidad se ha librado del papel de sirvienta de Ser, y ha asumido estado autónomo, incluso "constructivo". La naturaleza paradójica de las posiciones de partida elegidas de manera contingente le da a cada de estas distinciones iniciales su propia autonomía. $(2013,8)$.

Pero ésta y no otra es su dificultad epistemológica fundamental, la declaración que una teoría de la sociedad pueda librarse de compromisos ontológicos, lo que es muy diferente de afirmar que una característica básica de la ontología social es que se constituye epistémicamente (Venables 2016).

Para nosotros la observación es un proceso colectivo (de la comunidad científica) de indagación de la realidad (inagotable, compleja y cambiante) realizado a través del tiempo (depurado e históricamente perfectible). Desde este punto de vista, afirmar que el mundo es incognoscible con independencia del observador es plausible y obvio solo si definimos observación como una instantánea arbitraria del mundo. Definir los sistemas sociales como comunicaciones contingentes que actualizan una diferencia de sentido entre sistema y entorno, como plantea Luhmann, parece ser una ganancia en precisión teórica procedimental. Pero pensamos que el concepto de comunicación remite a un dato volátil autodefinido en blanco y, por tanto, teóricamente pierde posibilidades de ser ubicado y caracterizado empíricamente en cuanto a su procedencia (ontológicamente), así como tampoco es factible de ser clasificado y visualizado en una corriente general, como los hashtags, para ser comprendido (epistemológicamente) en un marco general (como el análisis de redes complejas concretas), que nos parece, es la manera como una ciencia fáctica realiza su tarea.

\section{Las incompatibilidades de la teoría con las explicaciones sociológicas}

Luhmann define la construcción teórica desde la epistemología y lo metodológico, cerrando la puerta a la discusión ontológica. Nosotros planteamos que este "salto ilegítimo" consiste en que su afán de argumentar a favor de una epistemología constructivista radical termina por plantear una ontología constructivista radical. Ese salto ilegítimo es lo que nos parece clave y problemático, porque entrega una solución equivocada a la necesaria y esquiva relación entre lo epistémico y lo ontológico en ciencia social, al intentar superar 
Fundamentos de la explicación en ciencias sociales: revisitando el constructivismo de Niklas Luhmann (20 años después)

Jorge Gibert Galassi; Juan Pablo Venables

esta relación sin considerar que "la ontología de lo social se constituye epistémicamente; vale decir, la pregunta acerca de qué es lo social implica inevitablemente la pregunta por las creencias que lo constituyen" (Venables 2016, 172).

Pero, más exactamente, ¿cómo la teoría de Luhmann impide las explicaciones sociológicas “corrientes"? A continuación, abordamos brevemente aquellos conceptos o propuestas que, a nuestro juicio, dificultan la explicación sociológica en la teoría luhmanniana

\subsection{Causalidad}

Las explicaciones sociológicas, como las de toda ciencia, lidian con la causalidad. En el caso de Luhmann, su método de las distinciones sería el protocolo convencional susceptible de repetirse para solucionar un problema de investigación científica, a saber, un vacío en el conocimiento científico o un vacío de explicaciones. El modelo deductivo-nomológico de la escuela analítica aboga por la determinación causal, y es más recurrente entre las técnicas experimentales. Claramente, éste no se asocia a la obra de Luhmann. Pero en el modelo inductivo, que se ocupa de las determinaciones no causales, hay variados tipos, como la estadística y otros ${ }^{3}$. Lo relevante sería que el método se orientara al descubrimiento o constatación de estructuras generales o de ley, no exclusivamente causales. Es decir, no determinar la causa de algo, no implica necesariamente que no exista explicación; podemos identificar el mecanismo de funcionamiento o las condiciones de ocurrencia de algo, como también otras opciones explicativas.

Luhmann, al proponer una teoría de la contingencia, en principio, no actuaría acorde al principio determinista, que es el instrumental metafísico de toda teoría científica. Además, exige "la elaboración de una teoría de la sociedad adecuada al objeto [...](lo que) hubiera exigido de ella incluirse a sí misma dentro de su objeto" (Luhmann 2006, 897), aun cuando explícitamente se argumenta que la novedad de su teoría es que prescinde de objetos.

Sin embargo, es su concepción de causalidad y argumento la que impide una explicación del todo robusta. En efecto, es plausible admitir que "la selección de los factores causales que deben tenerse en cuenta (y los que no) es cosa, pues, de observadores que emplean el esquema de la causalidad" $(2006,801)$, y que estos factores requieren decisiones de atribución. La discrepancia estriba en que no todos los observadores son iguales, del mismo modo que no concordamos con que la observación sea una operación individual inmediatista.

\footnotetext{
${ }^{3}$ Tanto en la determinación causal como no-causal, el principio determinista permite explicitar la teoría mediante situaciones donde sí X ocurre, entonces (necesariamente) Y siempre es/está producido/asociado por/a X.
}

Revista de Humanidades de Valparaíso, 2020, No 16, 191-214

(c) $(1)(9)$ CC BY-NC-ND 
Pero se desliza el reemplazo de "causa" por el de "atribución", uno sesgado desde el realismo, el otro, desde el constructivismo; por lo que toda nuestra crítica al constructivismo podría ser traspasada igualmente al concepto de atribución en tanto reemplazo del concepto de causa. En definitiva, no es posible hacer juicios verdaderos o falsos respecto de las relaciones entre causas y efectos (Luhmann, Vallejos y Estrada 2009).

Luhmann prefiere preguntar por cómo la causalidad puede ser observada (2009) desde una teoría de la construcción de semánticas sobre la causalidad que un sistema comunica a partir de la irritación del entorno, en vez de preguntar por las técnicas metodológicas de identificación de causas reales, agenciales o situacionales. Se concentra, obviamente, en la comunicación y, desde ahí, en las atribuciones causales de la comunicación. Esto es, en vez de adoptar el enfoque ontológico de agente-estructura, opta por contraponer la causalidad técnico-racional con la libertad individual (Luhmann 2009, 8-9).

No hay duda que la realidad social es semántica y que ésta es una de sus estructuras inter-subjetivas. Sin embargo, representación no es equivalente a semántica, toda vez que la primera supone ontología (ex ante), mientras que la segunda supone performatividad; intervención lograda o fallida (ex post). La primera describe, la segunda propone (sea efectivo/verdadero o inútil/falso)

\subsection{Enunciados y leyes}

Sin ontología no hay causalidad y sin causalidad, ¿qué tipo de enunciados puede sostener la teoría? Y si, acaso, puede hacerlo, ¿podrían algunos de éstos ser leyes sociológicas?

La construcción teórica que prescinde de seres humanos, en tanto entidades, plantea el problema de cómo vincular al "individuo" con un sistema social. Como se sabe, en la teoría de Luhmann los individuos están en el entorno del sistema social. La comunicación es una comunicación sin sujetos. ¿Cómo entonces, e.g., un sistema de interacción "arranca" y cómo se "mantiene funcionando" sin humanos, sin memoria? Luhmann apuesta a la abstracción: "El sistema de comunicación puede, pues, sustituir las prestaciones de memoria de sistemas psíquicos particulares mediante el uso de medios propios de comunicación; y puede finalmente crear con la escritura una memoria propia para sí" (Luhmann 2006, 54). Pero los libros no son, digamos, actantes; por lo que la respuesta nos parece insuficiente. Por el contrario, si pensamos que el elemento reproductor (o catalítico) de los sistemas son las estructuras de expectativas, los sistemas de interacción (humanos, ontologizados) tenderán a ser caracterizados con altos grados de estabilidad, orden, teleología y memoria; lo cual, por otra parte, podría ser más congruente con la evidencia empírica (Wang 2020).

Pero no se discutirá la categoría "sistema". Los sistemas existen y, como tales, poseen endoestructuras y exoestructuras especificadas como entidades reales (Bunge 2004). Si la autopoiesis es el mecanismo generador de los sistemas psíquicos y sociales mediante las 
comunicaciones, ¿cómo éstas se estructuran/funcionan y cuál es el problema teórico que resuelven? La respuesta de Luhmann son los medios de comunicación simbólicamente generalizados, que "resuelven el problema de la doble contingencia a través de la transmisión de la complejidad reducida" (Luhmann 1998a, 25). La comunicación política o económica reduce la complejidad del mundo mediante la distinción entre un adentro/afuera que otorga sentido y permite actualizar al sistema, e.g. sea el político o el económico.

Los medios de comunicación simbólicamente generalizados permiten entender la sociedad como un orden social emergente donde coexisten diferentes sistemas sociales que se acoplan estructuralmente de diferentes maneras, pues cada sistema es entorno de otro sistema. Así, "los acoplamientos estructurales deben entonces primero transformar las relaciones análogas en digitales si es que el entorno quiere influir en el sistema a través de ellas" (Luhmann 2006, 73).

Luhmann niega que la comunicación consista en una transferencia de conciencia a conciencia de "algo", de cierta entidad llamada "información" comunicada. Más bien, sólo se puede hablar de comunicación "cuando el cambio de estado del complejo A corresponde a un cambio de estado del complejo B, pese a que ambos complejos tengan otras posibilidades de definir su estado. Por lo tanto, comunicar-se significa limitar-se (poner límites a uno mismo y al otro)" (Luhmann 1991, 60). Tales límites se expresan debido a que la comunicación representa una síntesis de tres selecciones según Luhmann: información, notificación y comprensión o entendimiento. Sobre la base de tales selecciones, concluye que cada una de ellas resulta altamente contingente, pero efectiva a la hora de regular los límites. La comunicación adoptaría la forma de temas, que no es otra cosa sino sentido social tipificado. Ante la incertidumbre, surge un tema. Lo único que realmente comunica el sistema (psíquico o social) es la operación de distinción entre sistema y entorno. Lo que suceda con ella es mera atribución desde otro sistema que, al plantear así la cuestión, nuevamente, realiza su propia síntesis de comunicación. La pregunta, entonces, es: ¿hasta qué punto la teoría de la distinción, que es una teoría consagrada y validada en matemática y lógica, es pertinente para el caso de una ciencia factual? Pensamos que sí, pero desde el punto de vista de una teoría con una ontología social definida, que no es el caso bajo examen. De hecho, sus seguidores confirman que la teoría no está basada en ningún principio ontológico (Cadenas 2015, 210). La defensa de tal uso que hace Luhmann y la refutación de la teoría de los tipos como solución al problema de las paradojas en La ciencia de la sociedad (Luhmann 1996, 128-129) y en Teoría de los sistemas sociales II, a propósito del problema de la observación de estructuras latentes (Luhmann 1998b, 135-136) son controversiales e inverificables. Acá se afirma que lo central en disciplinas como la sociología es su contenido factual, por lo que la teoría de la distinción debería desempeñar un papel subordinado al primero.

¿Cómo el sistema supone al entorno al realizar la distinción entre sistema y entorno? Luhmann postula el concepto de acoplamiento estructural, que es requisito de la constitución autopoiética de la sociedad, caracteriza al sistema por estar operativamente clau- 
surado y cumple una función en los procesos de diferenciación social. El acoplamiento estructural surge, es un efecto, de la distinción entre sistema y entorno. Como tal, especifica las estructuras del sistema: "No determina lo que sucede en el sistema pero debe estar presupuesto, ya que de otra manera la autopoiesis se detendría y el sistema dejaría de existir. En este sentido, todos los sistemas están adaptados a su entorno (o no existirían)" (Luhmann 2006,73). Pero esto no impide la coevolución entre sistemas y entornos, mediante desacoples temporales entre sistemas de comunicación.

La interpenetración es un caso especial de acoplamiento estructural y Luhmann lo explica diciendo que:

no existe comunicación - de conciencia a conciencia- que no sea socialmente mediada, así como no existe comunicación entre individuo y sociedad [...] Sólo la conciencia puede pensar, pero no puede pensar con pensamientos propios dentro de otra conciencia. Y sólo la sociedad puede comunicar. En los dos casos se trata de operaciones propias de un sistema clausurado operativamente, determinado por la estructura $(2006,76)$.

El acople estructural entre sistemas psíquicos debe resolver que el "otro" aparece ante mí como una caja negra, por lo que la doble contingencia deberá ser transparentada y calculada por medio de algún mecanismo. A diferencia de Luhmann, que apuesta a la conducta propia basado en que es la única conducta por la que el sistema puede optar, ya que es la suya; nosotros asumimos lo mismo, pero en el sentido que ello es un efecto de estructuras de expectativas incorporadas. Esta forma es, también, mecanismo de selección de "lo que pueden observar" desde la dinámica particular de un sistema de interacción dado. Al seleccionar, dicha estructura genera una respuesta y de ese modo emerge un orden. Es decir, los participantes tienen la posibilidad de asegurar su propia conducta sólo en virtud de las estructuras de expectativas para tal o cual situación. El sistema de interacción se ha formado y delimitado por tales estructuras de expectativas. Por lo tanto, es la estabilización de las expectativas lo que permite la absorción de la incertidumbre, bajo la fórmula que la transforma de incertidumbre en transparencia y calculabilidad (Luhmann 1991, 126). En tal caso, la hipótesis es que las estructuras de expectativas y no las comunicaciones, serían los elementos del sistema de interacción.

Para todos los casos, lo relevante para Luhmann es cómo el sistema, que no es otra cosa que la distinción entre sistema y entorno realizada por el sistema, funciona de acuerdo a un círculo autorreferencial. La solución que da Luhmann no estriba en postular algún tipo de "hecho social", una dimensión objetiva (a lo Durkheim) a la que imputemos la función de proveedora de significación cosificada. Su solución es mucho más ambiciosa y se fundamenta en una teoría de la evolución social, donde se explicitan algunas "leyes" 
Fundamentos de la explicación en ciencias sociales: revisitando el constructivismo de Niklas Luhmann (20 años después)

Jorge Gibert Galassi; Juan Pablo Venables

de cómo esto ocurre, siendo la más importante la que dice que los sistemas coevolucionan entre sí. Nuestra diferencia con Luhmann es que todo proceso posee contenido sustantivo u objetivo, y no se restringe a la forma.

Y aunque Luhmann reconoce el "limitado valor explicativo del concepto de autopoiesis [...] Lo mismo en el caso de la comunicación: la sociedad se genera por la comunicación que presupone la operación autopoiética de la comunicación -pero de ahí no se desprende qué tipo de sociedad" $(2006,45)$, sigue pensando que "muestra con mayor precisión cómo la unidad puede ser reintroducida en sí misma mediante distinciones; y deja completamente abierta la cuestión de cuántas posibilidades existen y de si (y de qué manera) podrían coordinarse" $(2006,475)$. Por el contrario, acá se postula que al quedar indeterminado cualquier "hecho" causal, la explicación no logra constituirse como tal.

\subsection{Recursividad}

La recursividad enfatiza el hecho de que la comunicación para que sea tal, es decir, para que tenga sentido, debe ser seleccionada en una doble contingencia entre un ego y un alter o se acople estructuralmente un sistema psíquico con un sistema social o entre sistemas sociales. Lo interesante es que, en tal modelo, ningún elemento asume su producción: la comunicación sólo se debe a la comunicación, es autopoiética.

Hasta aquí, podemos identificar una teleología funcionalista y una argumentación circular. Es una teoría teleológica funcional porque busca en las funciones del sistema explicar para qué tal sistema es como es. Y la argumentación circular es la vía de respuesta: el sistema funciona para seguir siendo lo que es, sistema; y lo hace organizadamente. La falacia de este esquema es que, pensamos, la naturaleza de un sistema sólo puede ser definida parcialmente por sus funciones y ello, finalmente, suele terminar en la confusión entre sistema y función. La descripción en ese caso se vuelve siempre tautológica, como sugiere Provost (1985). Por ejemplo, Luhmann con la frase "la construcción del sistema y la diferenciación sistémica diferencian las posibilidades y aumentan las contingencias. Esto requiere la solución de los problemas de la selección por medio de mecanismos, es decir, medios que tienen que incorporarse a la estructura del sistema" (1998a, 54), está argumentando circularmente: la sociedad aumenta su diferenciación por los medios que han coadyuvado a su diferenciación en la medida que la sociedad se ha ido diferenciando.

Lo anterior se profundiza a propósito del problema del conocimiento: "conocimiento es lo que el conocimiento sostiene que es conocimiento" (Luhmann 1998b, 96). Si bien en muchas partes de su obra aboga por la verificación empírica de la teoría, esta frase expresa la ambivalencia de creer que la innovación en ciencia consiste en recombinar enunciados, en vez de crear y testear experimentalmente ideas. Confunde la comunicación científica con el conocimiento o, dicho de otra manera, plantea que el contenido de verdad de una proposición sólo se legitima socialmente. 
Fundamentos de la explicación en ciencias sociales: revisitando el constructivismo de Niklas Luhmann (20 años después)

Jorge Gibert Galassi; Juan Pablo Venables

\subsection{Equivalencia funcional}

El cuarto concepto a revisar es la crítica a la equivalencia funcional. Como se sabe, la solución a varios problemas respecto del alcance explicativo de los enunciados funcionalistas o teleológicos se fundó en la reducción al modelo nomológico. Esto se logra reemplazando la categoría de "función" por la categoría de "efecto", en aquellos casos en que una causa no implicaba más de un efecto; y en caso contrario, el concepto de función poseería mayor alcance explicativo, pero el dilema del límite -cuántos efectos son efectos o acaso existe alguna magnitud que implica empezar a pensar en equivalentes funcionales-lo tornaba problemático. Los funcionalistas como R. K. Merton (2002) y su famosa distinción entre funciones manifiestas y latentes, por ejemplo, abrieron la posibilidad de pensar que determinadas funciones sociales fueran desempeñadas por una multiplicidad de instituciones. La demostración de funciones unívocas sería imposible en la vida social $\mathrm{y}$, por lo tanto, el programa de reducir las explicaciones funcionales al modelo causal, inútil.

Luhmann captó brillantemente la oportunidad de radicalizar el análisis sociológico funcional. "Su verdadero sentido (habla de la función) consiste en situar la relación causal como contingente, es decir, exponerla a la comparación con otras posibilidades. Mediante la función se pueden buscar, entonces, posibilidades de sustitución y reconocer en sus condiciones de realización qué tanto o qué tan poco dependemos de ciertos arreglos causales" (Luhmann 1998b, 139). Pero una explicación que cambia el contenido sustantivo de sus enunciados es, con seguridad, una explicación problemática. Así, diremos que el argumento del equivalente funcional supone explicaciones arbitrarias. A diferencia de Luhmann, los autores realistas han acotado sus teorías, como es el caso de la teoría de la dependencia (Cardoso y Faletto 1969), acotada geográficamente; o la teoría del capitalismo tardío (Mandel 1975), acotada históricamente.

Para finalizar esta sección, diremos que el constructivismo epistémico o el "perspectivismo" en palabras de Searle (2001), es un planteamiento a tomar en cuenta, debido al sesgo teórico de toda observación. Pero el intento desontologizador de Luhmann termina por confundir los planos epistémico y ontológico. Un ejemplo de esta confusión entre el plano epistémico y el ontológico fue desarrollado en Venables (2016), y se observa claramente en el texto de Arnold (2008) acerca de los fundamentos del programa sociopoiético de Luhmann. Arnold señala que la epistemología constructivista ha "radicalizado la problematización acerca de los procesos que producen conocimientos, declarando que una realidad intrínseca e independiente del contexto que la indica es inalcanzable" $(2008,50)$, lo que implica una afirmación evidentemente epistémica, toda vez que hace referencia a la imposibilidad de acceder a la realidad con independencia del contexto observacional. Pero a renglón seguido deriva de lo anterior una consecuencia ontológica, cuando señala que "el constructivismo sostiene que la realidad se produce desde observaciones y no preexiste a ellas" (2008, 50). Esta afirmación es ontológica no en tanto contenido (no indica qué es esa realidad) sino en tanto origen o causa ontológica (se refiere a cómo es

Revista de Humanidades de Valparaíso, 2020, No 16, 191-214

(c) $(1)(9)$ CC BY-NC-ND 
que la realidad existe). Esta última idea entrega la que, a nuestro juicio, es la clave para desentrañar esta superposición no advertida entre los planos epistémico y ontológico: el constructivismo luhmanniano considera las afirmaciones ontológicas como afirmaciones de contenido, cuando lo cierto es que las afirmaciones ontológicas no tienen necesariamente que decir nada acerca de cómo es el mundo, sino sólo que éste tiene una manera de ser que es independiente de quienes la escrutan (Searle 1995; 2001; 2010). Así, con su intento desontologizador Luhmann evade el problema central: la relación entre lo epistémico y lo ontológico en ciencias sociales (Venables 2016).

\section{Potencialidades explicativas de la teoría luhmanniana: forzando un diálogo}

En esta sección se desarrollan algunos aspectos que consideramos promisorios para un diálogo fructífero de la teoría de Luhmann con la sociología contemporánea de corriente principal.

En su ya clásico Teoría general de los sistemas, Von Bertalanffy indicaba que una de las objeciones a la teoría de los sistemas es su falta de valor explicativo, pero contrargumentaba que hay grados en la explicación y que "en campos complejos y teóricamente poco desarrollados tenemos que conformarnos con lo que el economista Hayek llamó con justicia "explicación en principio"” $(1993,36)$.

El mismo Von Bertalanffy menciona numerosos ejemplos donde las aplicaciones de la teoría general de los sistemas pueden explicar y hasta predecir algunos fenómenos sociales. Pero advierte que ello requiere de un ejercicio absolutamente necesario de definir con rigor y precisión las entidades consideradas (1993, 204-206). Una teoría no es completa sólo porque posea una definición formalmente correcta de sus conceptos principales, inclusive si tal definición es lógica y matemática. En todo caso, es posible que muchas de las llamadas aplicaciones de la teoría general de sistemas correspondan a casos de modelos explicativos nomológicos o a explicaciones funcionales reducibles al modelo causal y en ningún caso analógico a una teoría de sistemas cerrados.

Se ha discutido la posibilidad que la teoría general de sistemas sea un conjunto isomorfo respecto a teorías particulares que utilizan el mismo enfoque, una especie de fractal. No hay duda alguna a que podemos encontrar analogías sorprendentes en distintos dominios de la naturaleza y entre éstos y los dominios de lo social. Pero aquí se plantean cuestiones de convención importantes. La primera es si vamos a considerar equivalentes a los términos "isomorfismo" y "analogía". La analogía, cuando no es suficientemente rigurosa, degrada en metáfora y. Por lo tanto, no es una posición adecuada. La segunda, es si vamos a definir isomorfismo de un modo tan estricto, es posible que eso impida el tratamiento de dos o más conjuntos reales desde un punto de vista comparado. En efecto, la idea que existe una correspondencia biunívoca que preserva una relación de orden entre dos conjuntos de enunciados (teorías) es un programa demasiado optimista. En cambio, 
Bertalanffy indica que dicho isomorfismo es posible, pero se refiere más bien a conjuntos de elementos empíricos (moléculas, personas, enzimas y muchas entidades más) cuya forma o dinámica expresa un orden o secuencia similar.

En ciencias sociales, una forma de hacer esto sería a la manera de Luhmann. Es decir, afirmando que existe un modelo genérico de funcionamiento de sistemas sociales y, acto seguido, formateando cada subsistema particular de acuerdo con esa configuración. Ello daría por resultado una teoría de sistemas sociales general que es isomórfica respecto de una teoría del sistema jurídico, educativo, político, etc. Habría que fundamentar en qué sentido las analogías son pertinentes, hasta qué punto las hipótesis auxiliares y las excepciones (como la teoría del sistema de interacción) tienen un tratamiento teórico adecuado y hasta qué punto muchos de los mecanismos que se postulan como funcionales a todos los subsistemas son mera especulación o soluciones simples a problemas complejos) ${ }^{4}$.

Sobre el problema del isomorfismo, habría que agregar una última afirmación. Como se decía, podemos constatar muchos isomorfismos, pero ellos se caracterizan por lo que vamos a denominar "de primer nivel", o sea, enunciados legales válidos en dominios muy distintos (como una función exponencial). Sin embargo, es muy difícil o imposible encontrar isomorfismos de segundo nivel, esto es, a nivel de teorías. La única solución sería restringir el concepto de teoría al de una ley simple, por ejemplo $\mathrm{y}=\mathrm{mx}+\mathrm{n}$, pero esta es una solución insostenible. Insistimos, la otra solución es la de Luhmann y que, a nuestro juicio, se basa en el precepto normativo de Von Bertalanffy (1993), o sea, la teoría general de sistemas como dispositivo regulador de la ciencia.

El tema de los límites es clave. Si no hay límites, sólo nos queda pensar que las definiciones conceptuales de una teoría adolecen de vaguedad intencional y extensional. O sea, la teoría tiene dificultades semánticas que pueden llegar a invalidarla. Por eso, la vigencia de toda teoría descansa en la correspondencia de sus afirmaciones con la evidencia empírica 5 .

La conexión de una teoría sistémica que incorpora al individuo permitiría, quizás, hacerla compatible con otras ideas particulares como el teorema de la profecía autocumplida, formulado originalmente como el teorema de Thomas). Otro tanto sucede con algunos enunciados legaliformes de la cibernética de sistemas, como que la retroalimentación (tanto negativa $=$ homeostasis, o positiva $=$ cambio) autorregula al sistema. En definitiva, el tratamiento de la complejidad de la relación entre sistema y entorno. Tanto la retroalimentación como las redundancias presentes en la comunicación de ambos sistemas

\footnotetext{
${ }^{4}$ Luhmann, en ocasiones, postula entidades concretas (el libro o el contrato) que participan en procesos o mecanismos concretos (la evolución social o el acoplamiento/simbiosis entre el sistema económico y el jurídico); y ello constituye un avance, pues permite orientar la verificación empírica.

${ }^{5}$ Para una parte de la comunidad de las ciencias sociales, esto es positivismo obsoleto. Pero si aceptamos que existen proxies metodológicos que objetivizan la subjetividad y los elementos intangibles de nuestra convivencia humana, no vemos necesario el animismo o la especulación decimonónica.
}

Revista de Humanidades de Valparaíso, 2020, No 16, 191-214 
Fundamentos de la explicación en ciencias sociales: revisitando el constructivismo de Niklas Luhmann (20 años después)

Jorge Gibert Galassi; Juan Pablo Venables

individuales que interactúan son restricciones, que demarcan el universo de posibilidades del decurso de la comunicación. Lo anterior quiere decir que los seres humanos procesan continuamente "el ruido" del entorno, ingresando al propio sistema como información lo necesario para mantener la retroalimentación necesaria para el sistema.

Las teorías deberían unificar leyes. Ciertamente, las teorías también deberían promover la generación de enunciados legaliformes. Si no tenemos teorías, no tiene importancia: los "grundisse" o "heurísticas" deberían permitir que los enunciados legales fueran incorporados de modo compatible con enunciados de otro tipo. O al menos, debería controlarse que no existiera incompatibilidad entre dichos enunciados.

Desde el punto de vista de las estrategias explicativas, ya es común avalar la tesis de la multidimensionalidad "blanda", tan característica del lenguaje de los cientistas sociales que al ser incapaces de someter a contrastación sus hipótesis, sólo se contentan con soluciones salomónicas que no dicen mucho, como la tesis que descansa en la noción de sentido común que en todo fenómeno social, quizás lo económico juega algún papel y quizás lo jurídico también o lo político, etc.; pero que jamás indica de qué modo y menos desarrolla una teoría sobre las relaciones conjeturadas entre tales dimensiones. Al respecto, Luhmann intenta superar estas limitaciones, mediante las categorías de código, programa, acoplamiento estructural, mecanismo simbiótico y otros. Esta opción podría tener validez en la medida que apuntara a una ontología social definida.

Planteado en términos de pretensión, ¿qué podríamos pedirle a la teoría? A lo menos dos cosas: i) que su contenido sea verificable y que la misma teoría abra caminos hacia esa dirección; y, ii) que los conceptos principales contenidos en las proposiciones y sus definiciones hayan sido dilucidados de tal forma que unos no estén implicados o contenidos en otros y que, en consecuencia, respeten los alcances y niveles de abstracción entre ellos mediante un análisis preciso de su consistencia; que sean formalmente correctas y materialmente (empíricamente) adecuadas. Los críticos, recalcitrantes y moderados, habitualmente han enfatizado la primera condición, sin preocuparse por la segunda (Viskovatoff 1999; Wagner 1997 y Zolo 1986). Satisfacer ambas condiciones es necesario, no sólo para la teoría en cuestión sino para las teorías sociales en general. Para la teoría de Luhmann en particular, esto es problemático, a pesar de su réplica hacia sus comentadores, donde ironiza respecto del rol de la investigación empírica y los califica de seguidores de una tradición caduca (Luhmann 1986). Se afirma aquí que no es posible calificar de caduca la pretensión de objetividad aunque, claro está, existen formas inadecuadas de pretender alcanzarla. En todo caso, está por verse si las críticas tienen sustento. 
Fundamentos de la explicación en ciencias sociales: revisitando el constructivismo de Niklas Luhmann (20 años después)

Jorge Gibert Galassi; Juan Pablo Venables

\section{A modo de conclusión}

De lo dicho hasta ahora, podrían plantearse tres reglas sobre lo que "no debe hacerse" y varias sugerencias que podrían ser útiles para revisitar de modo fructífero la explicación sociológica desde la perspectiva luhmanniana.

Primera regla: El "ismo", cualquiera que este sea, que no opera en base a la hipótesis de que existe un mundo real allá afuera, independiente de mi conciencia, es anticientífico (o no científico).

Segunda regla: El "ismo" que no opera a base de la hipótesis que conocer es trazar una distinción es anticientífico o, si se quiere, desconoce el sesgo teórico de la observación.

Tercera regla: El "ismo" que deduce que al aceptar la segunda regla se infiere la negación de la primera regla es anticientífico o, si se quiere, tiene problemas de "pensamiento arbitrario".

Lo que proponemos como sugerencias útiles son las siguientes:

Primera sugerencia: Siendo inevitable el uso de una distinción en toda observación científica $^{6}$, no debería extrañarnos que las ciencias sociales se desarrollen utilizando también esta operación.

Segunda sugerencia: Es decir, el uso de distinciones es inevitable tanto en la observación como en la exposición, pero cumplen roles distintos y se deben a diferentes exigencias según sean los objetivos de una u otra. Por ejemplo, la distinción en una exposición es una reconstrucción de lo real mediante cierta lógica argumentativa fundada en tales o cuales distinciones arbitrarias. Sin embargo, la distinción en la observación tiene un fundamento sustantivo. No distinguimos si existen elefantes que vuelan y elefantes que no lo hacen, distinguimos entre elefantes que viven en Asia y otros que lo hacen en África, por ejemplo. Esto nos lleva a la última sugerencia.

Tercera sugerencia: La ciencia busca u orienta su actividad hacia el descubrimiento y uso de distinciones sustantivas, reales, que permitan enriquecer, también, el contenido fáctico de los enunciados sobre la realidad objetiva, y sean refutables o verificables de algún modo. Cualquier distinción no es útil. Sólo lo es aquélla que sea compatible o pueda serlo con el requisito de describir, explicar y predecir eficientemente, entre otros varios requisitos que la comunidad científica exige a las distinciones usadas por los investigadores.

Dicho lo anterior, nos parece un momento apropiado para reflexionar sobre la teoría de sistemas sociales y su modalidad o enfoque explicativo.

\footnotetext{
${ }^{6}$ Obviamente, el concepto de observación con el cual trabajamos incluye la medición y el experimento.
}

Revista de Humanidades de Valparaíso, 2020, No 16, 191-214 
En particular, una de las deficiencias del enfoque explicativo en la teoría de sistemas sociales de Luhmann es su apuesta al isomorfismo entre la teoría general de sistemas sociales (sus "Grundrisse") y las teorías particulares (sobre el sistema educativo, el sistema político, etc.). Dicho sea de paso, apuesta "no jugada" en la mesa de juego de la ciencia. Se plantea que lo adecuado sería, por el contrario, testear tales lineamientos de legalidad y de explicación en los sistemas concretos, para saber cuáles son efectivamente "reales" y cuáles son reemplazables. En cualquier caso, valga la redundancia, hay que admitir que sin el trabajo monumental de Luhmann, no podríamos realizar jamás este ejercicio.

Pero este isomorfismo escabulle las conexiones entre los lenguajes auto-descriptivos de los sistemas, ya que los fenómenos concretos no se dejan reducir al lenguaje, conceptos y métodos de disciplinas particulares, sino que, por el contrario, la lógica investigativa se orienta a la búsqueda de explicaciones inter-nivel no reductivas. Ello quizás implique redefinir el corpus sustantivo de las ciencias sociales, para que deje de ser lo que es: una gran montaña de ideas económicas, sociológicas, culturales, politológicas, lingüísticas e históricas; sin suficientes vínculos sistemáticos.

Otra de las deficiencias del enfoque explicativo en la teoría de sistemas sociales de Luhmann es su enfoque funcionalista tout court. No hay novedad en dicha crítica (Rudner 1966). Lo único que podemos constatar es que nuestro autor podría haberse protegido contra ella si hubiera propuesto o hecho algún intento de confirmación de la teoría, como lo hizo Merton (2002) y varios otros sociólogos funcionalistas.

Más recientemente, la publicación científica Constructivist Foundations ha dedicado buena parte de su número 2 del 2015 a la teoría de Niklas Luhmann. En especial, se destaca el trabajo sobre autopoiesis de Cadenas y Arnold (2015a), que a nuestro juicio muestra una vez más que las dificultades de la teoría también se reproducen en los planteamientos al interior de la comunidad disciplinaria constructivista en sociología.

A la objeción que Urrestarazu plantea al trabajo de Cadenas y Arnold (2015a), de que ambos tergiversan el significado mismo del concepto de autopoiesis que Maturana y Varela (1995) crearon, se responde con que "sólo si se adoptara una postura ortodoxa, podría un uso no autorizado del concepto aparecer como $<$ injustificado $>$ o $<$ teoréticamente inadecuado>" (Cadenas y Arnold 2015b, 196). Pero esto podría ser interpretado así: si es heterodoxo, es teóricamente justificado, como si la novedad fuera un valor per se en detrimento de otros valores epistémicos como la rigurosidad o la verdad. Por otro lado, nada se dice respecto de si el uso ortodoxo posee valor de verdad o es correcto. Pero eso ya lo sabíamos, después de todo, la verdad no es muy importante desde el punto de vista sociológico constructivista (Gibert 2015). La opinión de Urrestarazu es que, para avanzar hacia una teoría constructivista, un mínimo a lograr es la fidelidad a los conceptos que han sido desarrollados por ella (Urrestarazu 2014). De otra manera, o Luhmann jamás debió haber importado el concepto o bien debió haberlo renombrado. Otro síntoma de la confusión y escasez de pertinencia es la respuesta de Cadenas y Arnold a la crítica de King 
(2014). Este último plantea la siguiente pregunta: ¿necesita la teoría de sistemas sociales de una teoría general de la autopoiesis? Y recibe la sorprendente respuesta de que no es necesaria... a lo que cabría agregar: entonces, ¿para qué se escribió el trabajo? No menos insólita es la oración seguida: "Por otra parte, eliminando cualquier referencia al concepto de autopoiesis en los trabajos de Luhmann, ello no conlleva una pérdida significativa" (Cadenas y Arnold 2015b, 197), lo que claramente se contradice con que los sistemas sociales según la teoría de Luhmann son sistemas autopoiéticos de comunicación (Rodríguez y Arnold 1991, 122 y ss.) y con su atribuida preocupación central por el carácter autorreferencial de los sistemas sociales (Cadenas, Mascareño y Urquiza 2012).

Consecuentemente, nuestra conclusión es que la dificultad epistemológica fundamental de Luhmann es su declaración de que una teoría fáctica (que de eso trata la sociología) puede librarse de compromisos ontológicos, pues, no hay manera de describir algo sin suponer una existencia o naturaleza de aquello que se describe.

En contraposición a las confusiones entre los continuadores de la obra luhmanniana, las críticas a la teoría y sus conceptos centrales han sido consistentes y diáfanos a través del tiempo (Zolo 1986; Wagner 1997; Viskovatoff 1999; Gibert y Correa 2001; Wan 2011; Tada 2020). Una muestra destacada de ello es el trabajo de Padgett y Powell, donde se critica que el concepto de autopoiesis de Maturana se reduce a su definición, pues "nada se dice en relación a la dinámica de cómo un sistema llega a ser tal" (2012, 56), un sesgo típico del programa científico cibernético. En ese sentido, se concluye que "Luhmann es más de lo mismo. Él enfatiza la clausura y la separación del mundo social humano del resto de la (manifestación de la) vida en vez de su interdependencia" (Padgett y Powell 2012, 57). Y terminan Padgett y Powell diciendo que la intuición de Luhmann les provoca simpatía, pues permite afirmar que un tipo de auto-catálisis distintivamente humana puede ser buscada en el "ámbito de la comunicación y el lenguaje" $(2012,57)$ siempre que la concibamos radicada en los contextos (reales) físicos y sociales abiertos en los cuales se usa.

En suma, a poco más de 20 años de la muerte de Luhmann, su obra y la de sus seguidores ya no pueden sustentarse en una promesa. La teoría ha gozado de un desarrollo suficientemente vigoroso como para estar presta a su confirmación, modificación o rechazo. Para ello, se propone, sería necesario ontologizar su teoría (Searle 1995), considerando a las comunicaciones como elementos estructurados y estructurantes (Archer 2009). Sobre el método, los seguidores del planteamiento luhmanniano podrían robustecer el método de construcción teórico, incorporando un código más amplio que el código binario de sistema/entorno; adentro/afuera; etc. Una manera de considerar los elementos es visualizándolos en sistemas que poseen una endoestructura y una exoestructura (resolviendo la solución formal de Luhmann respecto de la frontera entre sistema y entorno) y, finalmente, distinguiendo entre propiedades emergentes y resultantes, al interior de las propiedades de bulto (Bunge 2004), de modo que su teoría supere el atributo de contingencia y rescate el atributo de necesidad, que toda explicación incluye. 
Fundamentos de la explicación en ciencias sociales: revisitando el constructivismo de Niklas Luhmann (20 años después)

Jorge Gibert Galassi; Juan Pablo Venables

\section{Referencias bibliográficas}

Archer, M. (2009). Teoría social realista. Santiago de Chile: Ediciones Universidad Alberto Hurtado.

Arnold, M. (2008). La sociedad como sistema autopoiético: fundamentos del programa sociopoiético. En Osorio, F., Arnold, M., González, S., Aguado, E. La nueva teoría social en Hispanoamérica, pp. 46-81. Colección pensamiento universitario No 11. Toluca: UAEM.

Bryson, G. (2018). Cybernetics and contingency, codes and programs: an account of social system thinking in law and legal theory today. Doctorate of Philosophy (Ph.D.) in law, Birkbeck College, University of London.

Bunge, M. (1974). La ciencia, su método y su filosofía. Buenos Aires: Siglo Veinte.

Bunge, M. (1983). La investigación científica. Barcelona: Ariel.

Bunge, M. (1997). Epistemología. México DF: Siglo XXI.

Bunge, M. (1999). Las ciencias sociales en discusión. Buenos Aires: Sudamericana.

Bunge, M. (2004) Emergencia y convergencia. Novedad cualitativa y unidad del conocimiento. Barcelona: Gedisa.

Cadenas, H., Mascareño, A., Urquiza, A. (2012). Niklas Luhmann y el legado universalista de su teoría. Aportes para el análisis de la complejidad social contemporánea. Santiago de Chile: RIL Editores.

Cadenas, H. (2015). The reality of ontologies in Luhmann`s works. Constructivist foundations, 10(2), 210-211.http://constructivist.info/10/2/210.

Cadenas, H., Arnold, M. (2015a).The autopoiesis of social systems and its criticisms.Constructivist foundations, 10(2), 169-176.http://constructivist.info/10/2/169.

Cadenas, H., Arnold, M. (2015b). Author's response: On the criticisms against the autopoiesis of social systems. Constructivist foundations, 10(2), 196-198.http://constructivist. info/10/2/196.

Cardoso, F.H., Faletto, E. (1969). Dependencia y desarrollo en América Latina. México DF:Siglo XXI.

Demeulenaere, P. (Ed.) (2011). Analytical sociology and social mechanisms. New York: Cambridge University Press.

Elster, J. (1984). Marxismo, funcionalismo y teoría de juegos. Alegato en favor del individualismo metodológico. Zona abierta, 33, 21-62.

Fallding, H. (1976). Explanation theory, analytical theory, and the ideal type.EnK. Thompson, J. Tunstall (eds.), Sociological perspectives. London: Penguin books.

Gibert, J. (2006). La complejidad en ciencias sociales: ¿tema matemático, filosófico, científico o jerga posmoderna? Revista Integra, 10, 67-75.

Revista de Humanidades de Valparaíso, 2020, No 16, 191-214

(c) (1)@९ CC BY-NC-ND 
Fundamentos de la explicación en ciencias sociales: revisitando el constructivismo de Niklas Luhmann (20 años después) Jorge Gibert Galassi; Juan Pablo Venables

Gibert, J. (2015). Perspectivismo y verdad en sociología: Bourdieu y Giddens. Cinta de Moebio No 52, Marzo: 69-78. http://dx.doi.org/10.4067/S0717-554X2015000100006

Gibert, J., Correa, B. (2001). La teoría de la autopoiesis y su aplicación en las ciencias sociales. El caso de la interacción social. Cinta de Moebio, 12, 175-193.

Hempel, C. (1988). La lógica de la explicación científica. Barcelona:Paidos.

Joseph, J., Kennedy, S. (2000). The structure of the social. Philosophy of the social sciences, 30(4), 508-527. https://doi.org/10.1177/004839310003000402

King, R. D. (2014). Does social systems theory need a general theory of autopoiesis? Constructivist foundations, 10(2), 183-185. http://constructivist.info/10/2/183

Laurent, H. (1908). Statistique mathématíque. París: Octave doin editeur.

Luhmann, N. (1986). The theory of social systems and its epistemology: Reply to DaniloZolo's critical comments. Philosophy of the social sciences, 16(1), 129-134. https://doi. org/10.1177/004839318601600110

Luhmann, N. (1990). The cognitive program of constructivism and a reality that remains unknown. EnDelanty, G. y Strydom, P. (Eds.) (2003). Philosophies of social sciences. The classic and contemporary readings. Philadelphia: Open University Press.

Luhmann, N. (1991). Sistemassociales. México DF: Editorial Iberoamericana.

Luhmann, N. (1996). La ciencia de la sociedad. México DF: Editorial Iberoamericana.

Luhmann, N. (1998a). Teoría de los sistemas sociales. México DF: Editorial Iberoamericana.

Luhmann, N. (1998b). Teoría de los sistemas sociales II. México DF: Editorial Iberoamericana.

Luhmann, N. (2005). Confianza. U. Iberoamericana/Anthropos: Barcelona.

Luhmann, N. (2006). La sociedad de la sociedad. México: Herder.

Luhmann, N., Vallejos, A. y Estrada, M. (2009). Causalidad en el sur. Estudios sociológicos, 27, 3-29.

Mandel, E. (1975). Late capitalism. London: New Left Books.

Maturana, H., Varela, F. (1995). De maquinas y seres vivos. Santiago de Chile: Editorial Universitaria.

Merton, R. K. (2002). Teoría y estructura sociales. D.F. México: FCE.

Mill, J. S. (1882). A system of logic, ratiocinative and inducted. 8th Ed. New York: Harper \&Bros.

Osorio, F., Arnold, M., González, S., Aguado, E. (2008). La nueva teoría social en Hispanoamérica. Colección pensamiento universitario $\mathrm{N}^{\circ} 11$. Toluca: UAEM.

Padgett, J., Powell, W. (2012). The emergence of organizations and markets. New Yersey, USA and Oxford, UK: Princeton University Press.

Provost, W. (1985). Contingency and complexity in the social theory of NiklasLuhmann. International journal of general systems, 12(1), 39-53. https://doi.org/10.1080/03081078608934926

Revista de Humanidades de Valparaíso, 2020, No 16, 191-214

(c) (1)@९ CC BY-NC-ND 
Fundamentos de la explicación en ciencias sociales: revisitando el constructivismo de Niklas Luhmann (20 años después) Jorge Gibert Galassi; Juan Pablo Venables

Rasch, W. (2013). Luhmann's ontology. En la Cour, A. y Philippopoulos-Mihalopoulos, A. (Eds). (2013). Luhmann Observed. Radical Theoretical Encounters. Basingstoke, UK: Palgrave Macmillan.

Rodríguez, D. (2005). Nota a la versión en español. En N. Luhmann, Confianza, pp. VII - XXVII. U. Iberoamericana/Anthropos: Barcelona.

Rodríguez, D. (2006). La sociología y la teoría de la sociedad. En N. Luhmann, La sociedad de la sociedad, pp. IV - XXII. México: Herder

Rodríguez, D., Arnold, M. (1991). Sociedad y teoría de sistemas. Santiago de Chile: Editorial Universitaria.

Rudner, R. (1966). Philosophy of social science. Englewood cliffs: Prentice-Hall.

Schütz, A., Natanson, M. (ed.) (1962). Collected papers, I: The problema of social reality. The Hague: Martinus Nijhoff.

Schütz, A., Luckmann, T. (1973). Las estructuras del mundo de la vida. Buenos Aires: Amorrortu.

Searle, J. (1995). The construction of social reality. New York: The free press.

Searle, J. (2001). Mente, lenguaje y sociedad. Madrid: Alianza Editorial.

Searle, J. (2010). Making the social world. Oxford: Oxford University Press.

Tada, M. (2020). How Society Changes: Sociological Enlightenment and a Theory of Social Evolution for Freedom. The American Sociologist (online). https://doi.org/10.1007/s12108020-09464-y

Turner, J. (1991). Teorizar analítico. En A. Giddens, J. Turner et al. (Eds.) La teoría social, hoy, pp. 205-253.Madrid: Alianza editorial.

Urrestarazu, H. (2014). "Towards a consistent constructivist general systems theory". Constructivistfoundations, 10(2), 180-183. http://constructivist.info/10/2/180

Venables, J.P. (2016). Aportes para una ontología social realista. Cinta de moebio, 56, 172-186. https://doi.org/10.4067/S0717-554X2016000200005

Viskovatoff, A. (1999). Luhmann's theory of social systems. Philosophy of the social sciences, 29(4), 481-516. https://doi.org/10.1177/004839319902900402

Von Bertalanffy, L. (1993). Teoría general de los sistemas. México DF: FCE.

Wallace, W. (1983). Principles of scientific sociology. New York City: Aldine.

Wan, P. Y. (2011). (Re-)Problematizing the Luhmannian constructivist systems approach: A Bungean intervention. Current Sociology, 59(6),696-716. https://doi. org/10.1177/0011392111419756

Wang, Q. (2020). The cultural foundation of human memory. Annual Review of Psychology, 72 (online). https://doi.org/10.1146/annurev-psych-070920-023638

Wagner, G. (1997). The end of Luhmann's social system theory. Philosophy of the social sciences, 27(4), 387-409. https://doi.org/10.1177/004839319702700401

Revista de Humanidades de Valparaíso, 2020, No 16, 191-214

(c) $(1)(9)$ CC BY-NC-ND 
Fundamentos de la explicación en ciencias sociales: revisitando el constructivismo de Niklas Luhmann (20 años después) Jorge Gibert Galassi; Juan Pablo Venables

Zolo, D. (1986). Function, meaning, complexity: The epistemological premisses of Niklas Luhmann's sociological enlightenment. Philosophy of the social sciences, 16(1), 115-127. https://doi.org/10.1177/004839318601600109

Revista de Humanidades de Valparaíso, 2020, No 16, 191-214

(c) (1)@९ CC BY-NC-ND 